\title{
Postmenopausal adnexal torsion: rare case report
}

\author{
Ibrahim A. Abdelazim ${ }^{1,2}$, Mohannad AbuFaza², Yerbol Bekmukhambetov³, Gulmira Zhurabekova ${ }^{4}$, \\ Svetlana Shikanova ${ }^{5}$
}

1Department of Obstetrics and Gynecology, Ain Shams University, Cairo, Egypt

2Department of Obstetrics and Gynecology, Ahmadi Kuwait Oil (KOC) Company Hospital, Ahmadi, Kuwait

${ }^{3}$ Department of Oncology, West Kazakhstan Marat Ospanov Medical University, Aktobe, Kazakhstan

${ }^{4}$ Department of Normal and Topographic Anatomy, West Kazakhstan Marat Ospanov Medical University, Aktobe, Kazakhstan

${ }^{5}$ Department of Obstetrics and Gynecology No. 1, West Kazakhstan Marat Ospanov Medical University, Aktobe, Kazakhstan

\begin{abstract}
Most ovarian and/or adnexal torsions occur in reproductive age and are less common in postmenopausal age. A 49-year-old menopausal woman presented to the Emergency Department with abdominal pain. She had a palpable pelvi-abdominal mass and abdominal tenderness on examination. Departmental ultrasound and magnetic resonance imaging (MRI) showed a large multilocular right adnexal cyst $(15 \times 12 \mathrm{~cm})$ containing fluid with variable signal intensities on both $\mathrm{T} 1$ and $\mathrm{T} 2$ sequences (stained glass appearance) - most probably mucinous cystadenoma.

The studied woman signed an informed consent form and agreed to exploratory laparotomy and adnexectomy. After the pre-operative investigations, which were done according to the hospital protocol, including CA-125 (26 IU/ml) and anaesthesia consultation, she was scheduled for laparotomy.

At laparotomy an ovarian cyst originating from the right ovary was found with evidence of torsion of the infundibulopelvic and utero-ovarian ligaments (adnexal torsion). The right adnexa including the right ovary containing the ovarian cyst and the right fallopian tube was excised (adnexectomy). The histological examination of the excised adnexa confirmed the diagnosis of mucinous cystadenoma of the ovary. This report represents a rare case of an adnexal torsion in postmenopausal woman, to highlight that adnexal torsion can occur at any age and that the presence of ovarian mass or cyst predispose to adnexal torsion at any age.
\end{abstract}

Key words: postmenopausal, adnexal, ovarian, torsion, cystadenoma.

\section{Introduction}

Adnexal torsion is a rare emergency condition occurring at any age [1]. Adnexal torsion occurs in 2-15\% of women who have had previous surgical treatment for adnexal masses or cysts [1].

Most of the adnexal and/or ovarian torsions occur at reproductive age and are less common in postmenopausal age [1, 2].

This report represents a rare case of an adnexal torsion in a postmenopausal woman, to highlight that adnexal torsion can occur at any age and that the presence of ovarian mass or cyst predispose to adnexal torsion at any age.

\section{Case report}

A 49-year-old menopausal woman for the last two years presented to the Emergency Department of Ah- madi Hospital, Kuwait Oil Company (KOC) with abdominal pain. She had a palpable pelvi-abdominal mass and abdominal tenderness on examination.

Departmental ultrasound showed a large multilocular right adnexal cyst $(15 \mathrm{~cm} \times 12 \mathrm{~cm})$ with numerous thin septations, and the locules contained fluid of different echogenicity (mucin). Departmental ultrasound followed by magnetic resonance image (MRI) showed a large multilocular right adnexal cyst $(15 \mathrm{~cm} \times 12 \mathrm{~cm})$ containing fluid with variable signal intensities on both $\mathrm{T} 1$ and $\mathrm{T} 2$ sequences (stained glass appearance), most probably mucinous cystadenoma (Fig. 1).

The studied woman signed an informed consent form and agreed to exploratory laparotomy and adnexectomy. After the pre-operative investigations, which were done according to the hospital protocol, including CA-125 (26 IU/ml) and anaesthesia consultation, she was booked for laparotomy.

At laparotomy an ovarian cyst originating from the right ovary was found with evidence of torsion of the 


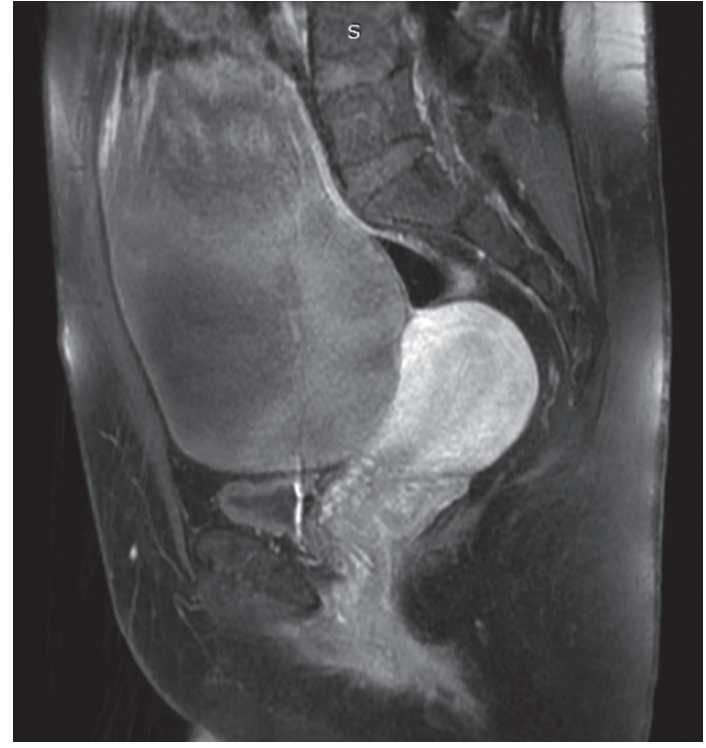

Fig. 1. Magnetic resonance image (MRI) of the studied pelvi-abdominal mass

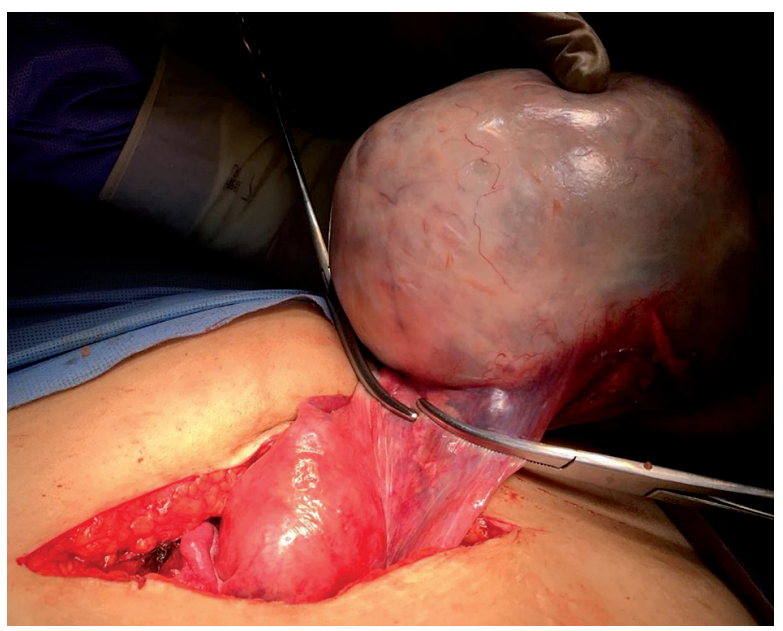

Fig. 3. Intra-operative image shows right adnexectomy including the right ovary containing the ovarian cyst and the right fallopian tube

infundibulopelvic and the utero-ovarian ligaments (adnexal torsion) (Fig. 2).

The right adnexa including the right ovary containing the ovarian cyst and the right fallopian tube was excised (adnexectomy) (Fig. 3).

The excised right adnexa including the ovarian cyst, which measured $15 \mathrm{~cm} \times 12 \mathrm{~cm}$, was sent for histological examination (Fig. 4).

The histological examination of the excised adnexa showed a multilocular cyst with variable size locules lined by flat columnar and low cuboidal cells overlying fibrous connective tissue stroma of spindle cells confirming the diagnosis of mucinous cystadenoma of the ovary.

The patient was discharged home after an uneventful post-operative hospital stay on the fifth post-operative day. Written consent from the studied woman and

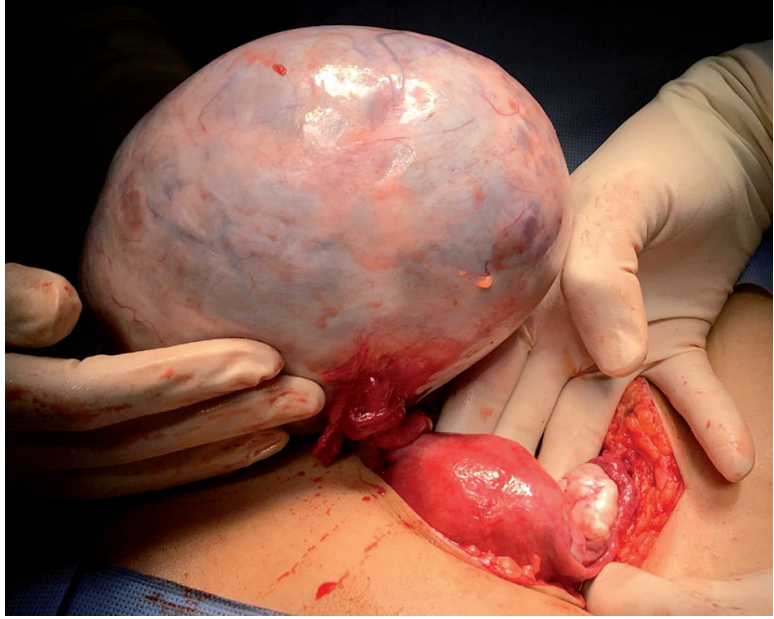

Fig. 2. Intra-operative image shows torsion of the right adnexa

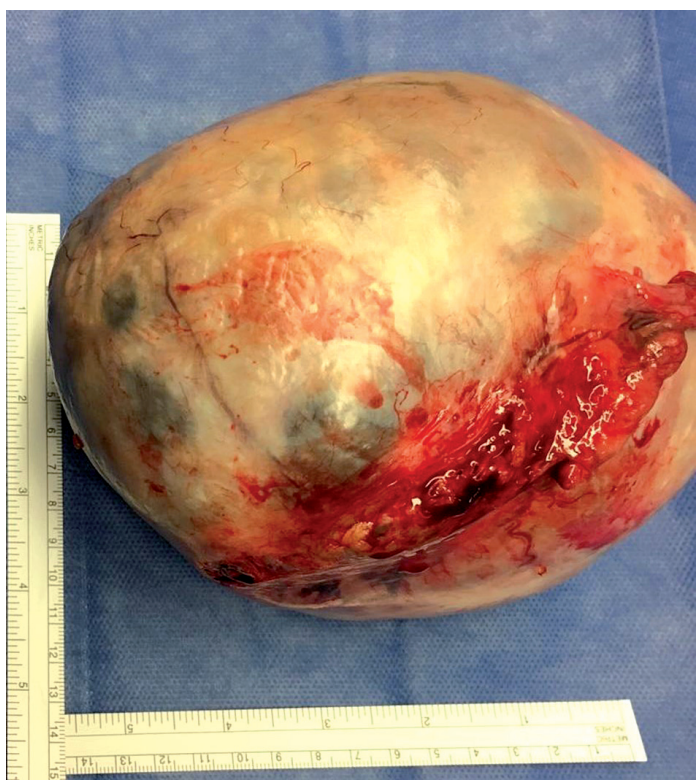

Fig. 4. Post-operative image of the excised adnexa including the ovary, containing the ovarian cyst and the right fallopian tube

departmental approval were taken to publish the studied woman's data as a case report.

\section{Discussion}

Mucinous cystadenoma of the ovary is a benign tumour from the surface epithelium of the ovary [3] and constitutes $15 \%$ of all ovarian tumours - usually presenting as a large multilocular cyst with smooth outer surface [3].

About $80 \%$ of the ovarian mucinous tumours are benign and usually present in reproductive age [1], rarely before puberty or after menopause [3].

The presence of large mucinous cystadenoma in the ovary (cyst or mass $>5 \mathrm{~cm}$ ) predispose to adnexal and/ or ovarian torsion [1]. 
The most common symptoms of adnexal and/or ovarian torsion are acute abdominal pain, nausea, and vomiting $[4,5]$.

Cancer antigen-125 (CA-125) is one of the tumour markers that should be tested to exclude malignancy or endometrioma in cases of suspected adnexal and/or ovarian masses [1].

Ultrasound is the first diagnostic tool in cases of suspected adnexal masses [1]. Magnetic resonance imaging (MRI) is helpful in diagnosing adnexal/ovarian torsion. In addition, the MRI can demonstrate more details regarding the components of the adnexal/ovarian mass [6].

Despite the development of several ultrasound models for the diagnosis of adnexal masses, the early diagnosis of ovarian cancers is still difficult. Moreover, identifying genetic risk alleles (germline BRCA1 and $B R C A 2$ mutations) for ovarian cancers has a significant impact on disease prevention strategies [7].

Froyman and Timmerman concluded that the International Ovarian Tumour Analysis (IOTA) Assessment of Different NEoplasias in the adneXa (ADNEX) model is clinically relevant and can give the predicted malignancy risk for different subtypes of adnexal masses [8].

Chen et al. concluded that the IOTA-ADNEX model showed good to excellent performance in distinguishing between benign and malignant adnexal masses in a Chinese setting [9]. In addition, Nowak et al. concluded that IOTA performance was highly satisfactory and similar to that of CA-125 in predication of malignancy risk for adnexal masses [10]. Both the IOTA model and CA-125 are complementary and can be used to differentiate between malignant and benign ovarian masses, although other clinical variables may also be important [10].

Once adnexal/ovarian torsion is suspected, surgery is the main treatment option [1]. In recent years, the treatment of adnexal/ovarian torsion has been evaluated, and ovarian conservative surgery including cystectomy and/or detorsion is recommended rather than salpingo-oophorectomy or adnexectomy [11, 12].

The studied woman presented with abdominal pain, nausea, vomiting, and abdominal tenderness, diagnosed as adnexal/ovarian torsion due to right-sided ovarian mucinous cystadenoma based on the ultrasound and MRI findings. The preoperative CA-125 was $26 \mathrm{IU} / \mathrm{ml}$. She was managed by exploratory laparotomy and right adnexectomy without ovarian conservative surgery based on her age (49 years old and menopausal for the last two years).

This report represents a rare case of adnexal torsion in postmenopausal woman to highlight that adnexal torsion can occur at any age and that the presence of ovarian mass or cyst predispose to adnexal torsion at any age.

\section{Conclusions}

The diagnosis of adnexal/ovarian torsion is challenging. Pelvic ultrasonography is the first diagnostic tool for diagnosis of adnexal mass or cyst, which predispose to adnexal torsion, while MRI is a complementary diagnostic tool to the pelvic ultrasound. Once adnexal/ ovarian torsion is suspected, surgery is the main treatment line using conservative ovarian surgery (ovarian cystectomy and/or detorsion) or radical ovarian surgery (oophorectomy or adnexectomy) according to the patient's age and fertility potential.

\section{Acknowledgments}

Authors are grateful to the studied woman who agreed for us to publish her data as a case report.

\section{Disclosure}

The authors report no conflict of interest.

\section{References}

1. Huang C, Hong MK, Ding DC. A review of ovary torsion. Ci Ji Yi Xue Za Zhi 2017; 29: 143-147.

2. Robertson JJ, Long B, Koyfman A. Myths in the evaluation and management of ovarian torsion. J Emerg Med 2017; 52: 449-456.

3. Kamel RM. A massive ovarian mucinous cystadenoma: a case report. Reprod Biol Endocrinol 2010; 8: 24.

4. Karaman E, Beger B, Çetin O, et al. Ovarian Torsion in the Normal Ovary: A Diagnostic Challenge in Postmenarchal Adolescent Girls in the Emergency Department. Med Sci Monit 2017; 23: 1312-1316.

5. Rey-Bellet Gasser C, Gehri M, Joseph JM, Pauchard JY. Is It Ovarian Torsion? A Systematic Literature Review and Evaluation of Prediction Signs. Pediatr Emerg Care 2016; 32: 256-261.

6. Naffaa L, Deshmukh T, Tumu S, et al. Imaging of Acute Pelvic Pain in Girls: Ovarian Torsion and Beyond. Curr Probl Diagn Radiol 2017; 46: 317-329.

7. Giampaolino P, Della Corte L, Foreste V, et al. Unraveling a difficult diagnosis: the tricks for early recognition of ovarian cancer. Minerva Med 2019; 110: 279-291.

8. Froyman W, Timmerman D. Methods of Assessing Ovarian Masses: International Ovarian Tumor Analysis Approach. Obstet Gynecol Clin North Am 2019; 46: 625-641.

9. Chen $H$, Qian L, Jiang $M$, et al. Performance of IOTA ADNEX model in evaluating adnexal masses in a gynecological oncology center in China. Ultrasound Obstet Gynecol 2019; 54: 815-822.

10. Nowak A, Soja M, Masternak M, et al. Evaluation of adnexal tumours in the International Ovarian Tumor Analysis system in reference to histopathological results. Prz Menopauzalny 2019; 18: 141-145.

11. Hubner N, Langer JC, Kives S, Allen LM. Evolution in the Management of Pediatric and Adolescent Ovarian Torsion as a Result of Quality Improvement Measures. J Pediatr Adolesc Gynecol 2017; 30: 132-137.

12. Adilgereyeva AS, Abdelazim IA, Zhurabekova GA. Clinical and pathological features of women with adnexal masses admitted as emergency cases to the Gynaecology Department of West Kazakhstan University. Prz Menopauzalny 2019; 18: 180-183. 